\title{
Research in Prehospital and Disaster Health and Medicine: the Manuscript Methods Section
}

\author{
Samuel J. Stratton, MD, MPH
}

This Editorial is another in a series published to support Disaster Health and Medical Research. ${ }^{1-5}$ This series of papers provides authors with the guidelines used by Prehospital and Disaster Medicine (PDM) for selecting and publishing manuscripts. Most important, the series is designed to provide new researchers with direct information on planning studies and preparing manuscripts. Experienced researchers are encouraged to comment and criticize the series presentations to help develop the field of Disaster Health and Medical Research.

The ability of credible health and medical journals to accept submitted manuscripts is limited. Originally, the acceptance of manuscripts was limited by the number of print publication pages available to a journal. In the current era of electronic publishing, the limiting factor for acceptance of manuscripts has evolved to the restrictive cost and availability of copy-editing means to prepare manuscripts for publication. Reputable journals place considerable resources into proper formatting and editing (copy-editing) of manuscripts to assure readability and standardized formatting for journal indexing services.

To establish priority for publication of manuscripts, scientific journals rely on expert peer-review (preferably blinded). It has been the experience for PDM that concern expressed by peerreviewers regarding study design and methods is the most frequent reason for rejection of a manuscript for publication. Many experienced section editors and reviewers will first consider the Methods section of a paper when considering a manuscript for publication.

The Methods section serves a number of requirements for a scientific manuscript, including:

1. The Methods section describes the process followed by the researcher in such a way that another researcher can reproduce the study to confirm the results of the original study. This is a foundation of the scientific method: that of reproducibility of results of a study.

2. The Methods section provides a description of the study design, including the study population, study outcome, and study data source. This information provides readers with a precise understanding of what is being studied.

3. The Methods section provides definitions of study variables which allows for correct understanding of what is being studied.

4. The Methods section describes the statistical or operative mechanics by which the study data are tested to make valid inferences and conclusions relative to the study objective.

As can be determined by the items listed above, the Methods section of a manuscript is a detailed description of how a study was conducted. The Methods section should be written in a basic manner that can be understood by any knowledgeable reader.

Reputable health and medical journals adhere to the guidelines of the International Committee of Medical Journal
Editors (ICMJE). ${ }^{6}$ These guidelines provide recommendations for writing a Methods section. An important standard described by the ICMJE is that the Methods section should only contain information related to the planning and development of the study protocol. Information obtained during the conduct of the study is more appropriately presented in the manuscript Results section. In addition, the Methods section should define the study population, with an explanation of the subjects included in the study and those excluded. Study variables that define the study subjects, such as age or gender, should be explained when they help identify a specific study population. In general, all study variables are to be defined, particularly those that may be vague or confused such as the terms "race" or "ethnicity."

The technical information provided in the Methods section should be clear and sufficient enough for others to reproduce the study exactly as done for the research reported in the manuscript. For studies that rely on secondary data, such as comprehensive reviews, the techniques used for locating the data should be reported, as well as the methods used to select data sources and to extract and synthesize the data used to report findings or conclusions. Specific standards exist for methods that use special types of data sources such as surveys, databases, and medical chart reviews. $^{7-10}$

Qualitative research designs generally should follow the guidelines for a Methods section as described above. Important for qualitative studies is that any pre-structuring of the study is described in the Methods section, and if the study was not prestructured, that be explained. Any particular phenomena studied should also be identified. How relationships with study subjects were developed to generate study data is important to describe and if actions were taken to minimize the limitations of study relationships, they should be described. Sampling decisions for qualitative studies are important to describe; while most qualitative research is done using convenience sampling, it is still important to note this in the manuscript Methods section. The qualitative data collection plan should be described and any methods used for confirmation of data (such as triangulation techniques) should be reported clearly.

The statistical analysis planned prior to conduct of the actual data collection is described in the Methods section. Unplanned secondary data analysis (for example subgroup analysis) is more appropriately described in the Results section and is discouraged in quantitative studies as it can lead to bias in results. In addition to standard statistical analysis, when appropriate, the sample size required to complete the study should be estimated prior to initiation of data collection. A common mistake made in reporting statistical tests is to neglect to state which statistical test is applied to which data. While usually obvious in the description of the results of a study, there is still a need to identify the statistical test associated to specific data. Equally important is to identify all variables that are included in multi-variable analysis, either in the 


\section{Checklist for the Basic Elements of a Manuscript Methods Section}

Statement of study design, including if prospective or retrospective.

$\square$ Provide reference citations if specialized or novel study design was used.

Description of study population, including inclusion and exclusion criteria.

State the study intervention or variables of interest (depending on study design).

Identify and define the outcome(s) of interest.

Study time frame.

Define variables used or identified in the study.

Identify study data source:

$\square$ Define data elements or provide data dictionary as an appendix.

$\square$ Identify how data were obtained, extracted, or coded and maintained.

$\square$ Identify how data were validated for accuracy.

Identify how missing data were managed.

$\square$ Identify statistical methods used for each form of data used in the study:

$\square$ Include description of significant probability value if hypothesis testing (P values) used; usually, $\mathrm{P} \leq .05$ for two-tailed analysis.

$\square$ Include measures of precision (confidence intervals, standard deviation, or quartiles) when used.

$\square$ For multi-variable analysis methods, identify all variables used.

For qualitative studies, define how relevance of data was determined.

Define statistical terms, abbreviations, and symbols when used.

$\square$ Identify statistics computer software when used.

$\square$ Provide estimate of number of subjects needed to study for clinical significance or, for qualitative studies, how data were determined to be valid (such as theme saturation).

$\square$ For human-subject-based studies (including surveys), state if study was reviewed by a human subjects research ethics committee (state name of committee) or describe in detail how the Declaration of Helsinki was applied to the research.

Do not include results or discussion in the Methods Section.

Table 1. Methods Section Checklist

Stratton @ 2016 Prehospital and Disaster Medicin

manuscript text or by table. While probability testing (generating a $P$ value) is often used to infer statistical significance, equally important is to design studies where precision measures (confidence intervals, standard deviation, or quartiles) are provided for measures of central tendency (means and medians). Standard practice is to specify any computer software used in conducting statistical analysis.

Providing study design organization charts for mixed method (quantitative and qualitative) or other complex study designs helps readers understand the flow of a study. In addition, reference citations should be provided when specialized study methods are used that are not commonly described in general statistical reference texts.

When appropriate, the review of a study protocol by an independent human subjects' protection committee (Ethics Committee or Institutional Review Board) should be noted at the end of the Methods section. The name of the committee should be provided along with the protocol review number and committee determination of status (approval, exemption, review declined, or waiver of informed consent). When an author does not have access to a human subjects' protection committee, the study should be conducted in accordance with the Declaration of Helsinki ${ }^{11}$ with a detailed description of how this was accomplished.

A checklist for writing a Methods section for PDM is provided in Table 1. This checklist provides the essential elements for a Methods section. In addition to the essential elements of a Methods section, authors should provide any further information that will clarify study design and methods.

In summary, the Methods section is an important and essential element of a scientific manuscript. It supports the validity of a study and allows the reader an understanding of the critical elements of a study. Importantly, the Methods section supports the scientific method by allowing others to repeat a study to show that the results of the original study are reproducible. 
References

1. Stratton SJ. Field Reports: can they add to the prehospital and disaster knowledge base? Prehosp Disaster Med. 2015;30(5):437.

2. Stratton SJ. Assessing the accuracy of survey research. Prehosp Disaster Med. 2015; 30(3):225-226

3. Stratton SJ. Research in prehospital and disaster health and medicine: the Introduction section of a study manuscript. Prehosp Disaster Med. 2014;29(5):439-440.

4. Stratton SJ. Research in prehospital and disaster health and medicine: developing a research objective statement. Prehosp Disaster Med. 2014;29(4):341-343.

5. Stratton SJ. Publishing survey research. Prehosp Disaster Med. 2012;27(4):305.

6. Preparing for submission. International Committee of Medical Journal Editors Web site. http://www.icmje.org/recommendations/browse/manuscript-preparation/preparing-forsubmission.html. Accessed December 15, 2015.

7. Kaji AH, Schriger D, Green S. Looking through the retrospectoscope: reducing bias in emergency medicine chart review studies. Ann Emerg Med. 2014;64(3):292-298.
8. Stratton SJ. Using pre-existing databases for prehospital and disaster research. Prehosp Disaster Med. 2015;30(1):1-3.

9. Worster A, Haines T. Advanced statistics: understanding medical record review (MRR) studies. Acad Emerg Med. 2004;11(2):187-192.

10. Gilbert EH, Lowenstein SR, Koziol-McLain J, Barta DC, Steiner J. Chart reviews in emergency medicine research: where are the methods? Ann Emerg Med. 1996;27(3): 305-308.

11. World Medical Association Declaration of Helsinki Ethical Principles for Medical Research Involving Human Subjects. World Medical Association Web site. http:// www.wma.net/en/30publications/10policies/b3/. Accessed December 15, 2015.

Online publication: January 4, 2016

doi:10.1017/S1049023X15005592 